\title{
A Survey of Mosquito Species in Public Schools of Metro Manila, Philippines Using Ovitraps as Surveillance Tool
}

\author{
Myra S. Mistica, ${ }^{1}$ Virginia R. Ocampo, ${ }^{2}$ Lilian A. De Las Llagas, ${ }^{1}$ \\ Arlene G. Bertuso, ${ }^{1}$ Fe D. Alzona ${ }^{2}$ and Ester A. Magsino ${ }^{2}$ \\ ${ }^{1}$ Department of Parasitology, College of Public Health, University of the Philippines Manila, Ermita, Manila, Philippines \\ ${ }^{2}$ Crop Protection Cluster, College of Agriculture, University of the Philippines Los Baños, Los Baños, Laguna, Philippines
}

\begin{abstract}
Objective. This study assessed the species composition of mosquitoes collected from ovitraps in selected public schools of Metro Manila, Philippines.

Materials and Methods. Ovitraps were installed from November 2013 to February 2014, in purposively selected public elementary and high schools of Metro Manila. Ovitraps were installed inside classrooms and collected weekly and examined for mosquito eggs and larvae. Speciation was done once a month from November 2013 to February 2014. All positive paddles were immersed in water for larval emergence, reared to fourth instars and were identified using taxonomic keys.
\end{abstract}

Results. A total of 1,482 ovitraps were installed in the selected public schools, and 18,325 larvae were collected from elementary schools, while 16,670 larvae were collected from high schools.The mean ovitrap index or infestation rate was $49.69 \%$ during the period of data collection. Mosquito larvae were identified as Aedes aegypti (88.94\%) and Aedes albopictus (11.06\%). Aedes aegypti and Aedes albopictus coexisted in some of the examined paddles. No significant differences were observed between the type of school and the presence of Aedes larvae. Significant differences were observed between total number of mosquito larvae and months of collection.

Conclusion. The presence of Aedes mosquitoes in schools evidently shows that continuous vector surveillance in schools is necessary as this information will help in the formulation of proactive vector control activities, thereby preventing the occurrence of mosquito-borne diseases.

Key Words: mosquito, Aedes, vector, school, surveillance

\section{INTRODUCTION}

Mosquitoes are known vectors that transmit pathogenic microorganisms contributing to the toll of morbidities and mortalities worldwide. These organisms proliferate in the environment and continually affect the quality of life of each individual affected by mosquito-borne diseases. In the

Poster presented at the Annual Scientific Conference of the Philippine Association of Entomologists, Inc. during the $45^{\text {th }}$ Annual Convention of the Pest Management Council of the Philippines, Inc., May 5 - 8, 2014, Cebu City.

Corresponding author: Myra S. Mistica, MPH

Department of Parasitology

College of Public Health

University of the Philippines Manila

625 Pedro Gil St., Ermita, Manila 1000, Philippines

Telephone/Fax no.: +632 5235929

Email:msmistica@up.edu.ph
Philippines, numerous mosquito-borne diseases like dengue, malaria, and filariasis continue to affect each individual. Newer reports present that mosquitoes likewise transmit diseases like the Chikungunya and Zika viruses. The burden of mosquito-borne diseases to the general public may bring about morbidity and or death.

Among the mosquito-borne diseases, dengue is considered to be the most important disease in the world ${ }^{1}$ and in the country. Millions of lives worldwide are at risk of the viral infection transmitted by mosquito vectors. All 
the regions of the country appear to be vulnerable to the transmission of mosquito-borne diseases. Dengue infections are one of the important mosquito-borne diseases, as 186,416 cases and 59 deaths were reported in 2012. In Metro Manila alone, a total of 16,046 cases and 29 deaths have been documented. ${ }^{2}$ The same report presents that children mostly under 10 years of age are affected. Like dengue, the Chikungunya viral infection is an emerging concern in the country. As of 2013, a total of 4,745 cases of the viral infection were reported, and $47.03 \%$ were serologically confirmed. Of this total number, about 674 cases, with $59.40 \%$ serologically confirmed, were reported in Metro Manila. ${ }^{3}$ Both Aedes aegypti and Aedes albopictus have been known to transmit mosquito-borne diseases. The occurrence of Aedes mosquitoes in the country has been documented nationwide, making all regions of the country vulnerable to the transmission of mosquito-borne diseases; ${ }^{4,5,6}$ however, limited studies on the occurrence and population ecology of Aedes albopictus in the country have been reported.

Different localities in the country recognize the threats brought about by these diseases and consider different strategies in looking after the welfare of the general population. Children in schools are recognized as important vulnerable groups in the Philippines, as their burden of mosquito-borne diseases is great as they may be hospitalized, suffer long-term disabilities, incur foregone opportunities by missing out in school, and, in worse conditions, even die. The risk of being bitten by mosquitoes among children is very high, especially that mosquitoes can thrive abundantly in environments as long as artificial containers can act as their breeding habitats. ${ }^{7}$ There is a need to monitor the occurrence of mosquito vectors in places where children meet and congregate and to institute vector surveillance strategies so that appropriate preventive and control measures may be put in place to protect the children's public health and welfare.

Most vector surveillance activities in the country have been directed in the communities, and to date, no study has explored vector surveillance activities in populous places like schools. This study aimed to assess the species composition of mosquitoes collected from ovitraps in selected public schools in the National Capital Region in the Philippines. Ovitraps are proven to be sensitive in detecting mosquitoes like the Aedes populations even in low infestation levels and in indoor and outdoor environments. ${ }^{8}$ Results pertaining to this study are significant, as they enable us to understand the vector's dynamics and help government and communities in formulating comprehensive vector control operations in public spaces like schools.

\section{MATERIALS AND METHODS}

The study was conducted in selected public elementary and high schools located in the 16 cities and 1 municipality of Metro Manila. Based on the population per city/ municipality, one study site was assigned for every 500,000 individuals. The study sites were the barangays (villages) in the city/municipality. A total of 28 barangays were drawn out. In every barangay, 1 public elementary and 1 public high school were selected. The selection of the schools was based on the student enrollment population within that barangay. In the case of more than one public high school or public elementary school in the barangay, the school with the most number of students was chosen. The public elementary and public high schools in the study sites were determined based on the population size of the city and municipality of Metro Manila. A total of 56 public schools were identified. Twenty-nine elementary schools and 27 high schools were purposively selected from the different communities of Metro Manila depending on the student enrollment population data from the Department of Education. The total number of ovitraps installed was based on the total number of instructional classrooms in each school. An instructional classroom was defined as a room with walls and roof, has no air-conditioned facility and is used to hold the classes from $8 \mathrm{AM}$ to $5 \mathrm{PM}$. Arbitrarily, $30 \%$ of the total number of instructional classrooms was used as fixed collection sites. A total of 735 ovitraps were installed in the elementary schools, and 747 ovitraps were installed in the high schools. The ovitraps were placed inside the classrooms in areas free from disturbances. A sign was placed near the ovitraps to inform the students not to disturb or play with the ovitraps. The trained field collectors installed and collected the ovitraps every week from November 2013 to February 2014. During collection, it was ensured that the ovitrap cans were thoroughly cleaned by brushing the inside surface, then refilled with clean tap water and new paddles for re-installation. Collected water and paddles were brought to the Medical Entomology Laboratory of the Department of Parasitology, College of Public Health, UP Manila. The collected water was examined for larvae, and paddles were examined for the presence of eggs weekly. Speciation was done once a month from November 2013 to February 2014. All positive paddles were individually placed in plastic containers with water and small amounts of ground dog food and left to stand for $4-5$ days to allow the development of the emerged larvae to the fourth instar stage. The fourth instar larval stages were taxonomically identified using standard entomological keys. ${ }^{9}$ Mosquito larvae per location, month of collection, and type of school (elementary or high school) were recorded and compared using analysis of variance (ANOVA) and independent $t$ test. A test with a $p<0.05$ indicates that all statistical analyses are significant. All statistical analyses were performed using the GNU PSPP software.

\section{RESULTS}

A total of 1,482 ovitraps were installed in the selected public schools. From November 2013 to February 2014, a 
total of 18,325 (52.36\%) mosquito larvae were collected from public elementary schools and 16,670 (47.64\%) larvae from public high schools of Metro Manila, Philippines. During this period, the mean ovitrap index or infestation rate was $49.69 \% \pm 22.1$. From the collected larvae in elementary schools, 16, $214(88.48 \%)$ were identified as Aedes aegypti, and 2,111 (11.52\%) were Aedes albopictus. In public high schools, 14,909 (89.44\%) were identified as Aedes aegypti and 1,761 (10.56\%) were Aedes albopictus. Most of the collected mosquito larvae were those of Aedes aegypti (88.94\%), compared to Aedes albopictus (11.6\%). Figure 1 shows the distribution of Aedes spp larvae in the selected public schools. Each colored dot also represents the selected schools in the study. It can be noted that in most schools, the co-existence of both Aedes species were documented.

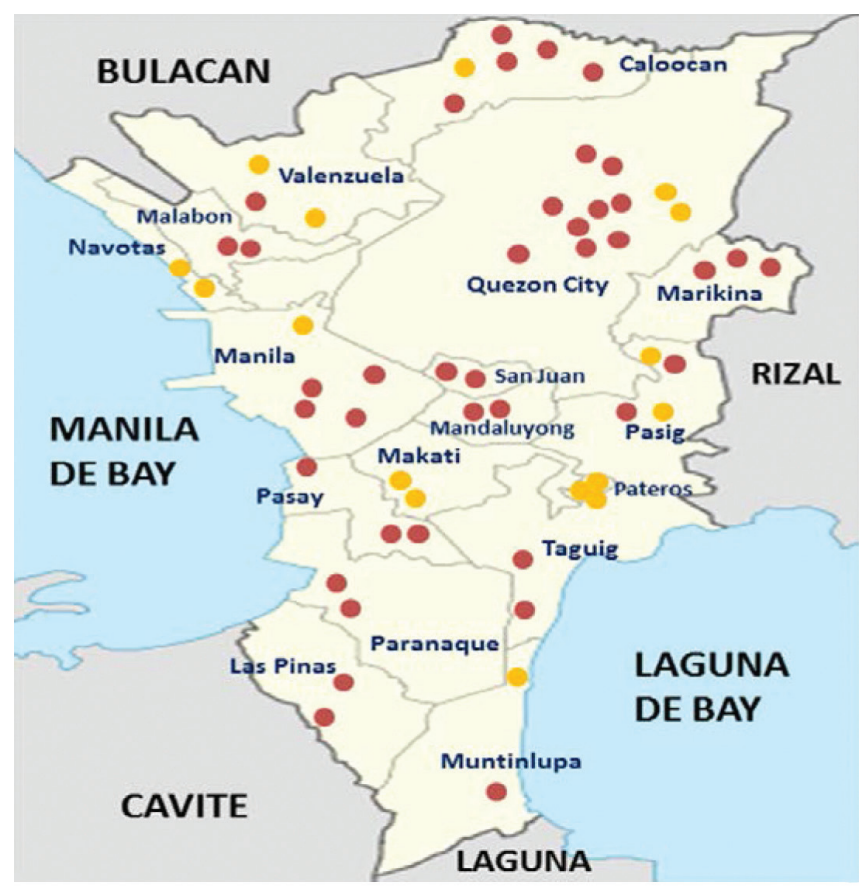

LEGEND: Ae. aegyptiand Ae. albopictus Ae. aegyptionly

Figure 1. Distribution of Aedes spp larvae in selected public schools in the NCR.
The Aedes aegypti fourth instar larva's abdomen shows comb scales with stout, subapical spines, and/or multiple stout spines, whereas the Aedes albopictus fourth instar larva's abdomen shows comb scales without subapical spines or multiple stout spines (Figure 2).

Nineteen of the 29 elementary schools and 21 of the 27 high schools had mixed Aedes infestations in the ovitraps examined. Single infestation of Aedes aegypti in the ovitraps of nine elementary and six high schools were observed. No single infestation of Aedes albopictus in the ovitraps of the public elementary and high schools of Metro Manila was seen. There was no significant differences on the type of school and the total mosquito larvae $(t=0.206, p=0.837)$.

No significant differences on the type of school and the Aedes aegypti larvae $(t=0.388, p=0.698)$ alone were seen. Likewise, no significant differences on the type of school and the Aedes albopictus larvae $(t=-0.404, p=0.686)$ alone collected were seen. Figure 3 shows that Aedes spp larvae were found in all selected schools from the sixteen (16) cities and one (1) municipality of Metro Manila. No significant differences were observed on Aedes aegypti larvae seen across all the cities and municipality assessed $(F=0.811, p=0.674)$. Significant differences of the total mosquito larvae $(F=5.978, p=0.000)$ and Aedes albopictus larvae $(F=7.824, p=0.000)$ based on the different cities and municipality assessed were observed. Significant differences on the total mosquito larvae $(F=8.396, p=0.000)$, Aedes aegypti larvae $(F=7.428, p=0.00)$, and Aedes albopictus larvae $(F=3.166, p=0.001)$ across the months of collection were likewise observed, as shown in Figure 4.

\section{DISCUSSION}

Dengue fever and Chikungunya infections are important causes of morbidities in the country. ${ }^{3}$ The occurrences of these ailments have been attributed to the mosquito vectors, and reports have indicated that the most commonly inflicted are children below 15 years of age. ${ }^{10}$ Most vector surveillance studies in the country have been focused in the community setting. This was the first study that focused on vector surveillance in a school setting. This was a prospective study,
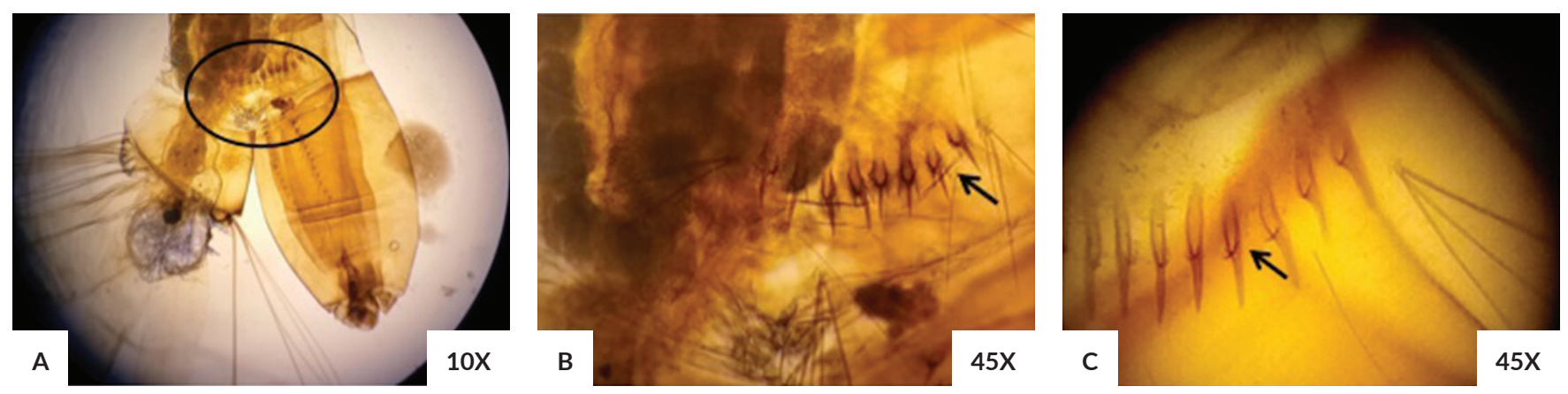

Figure 2. Taxonomic characters of the fourth instar larval stages of Aedes aegypti and Aedes albopictus. (A) Abdomen with comb scales. (B) Comb scales with stout subapical spines Aedes aegypti. (C) Comb scales without subapical spines Aedes albopictus. 


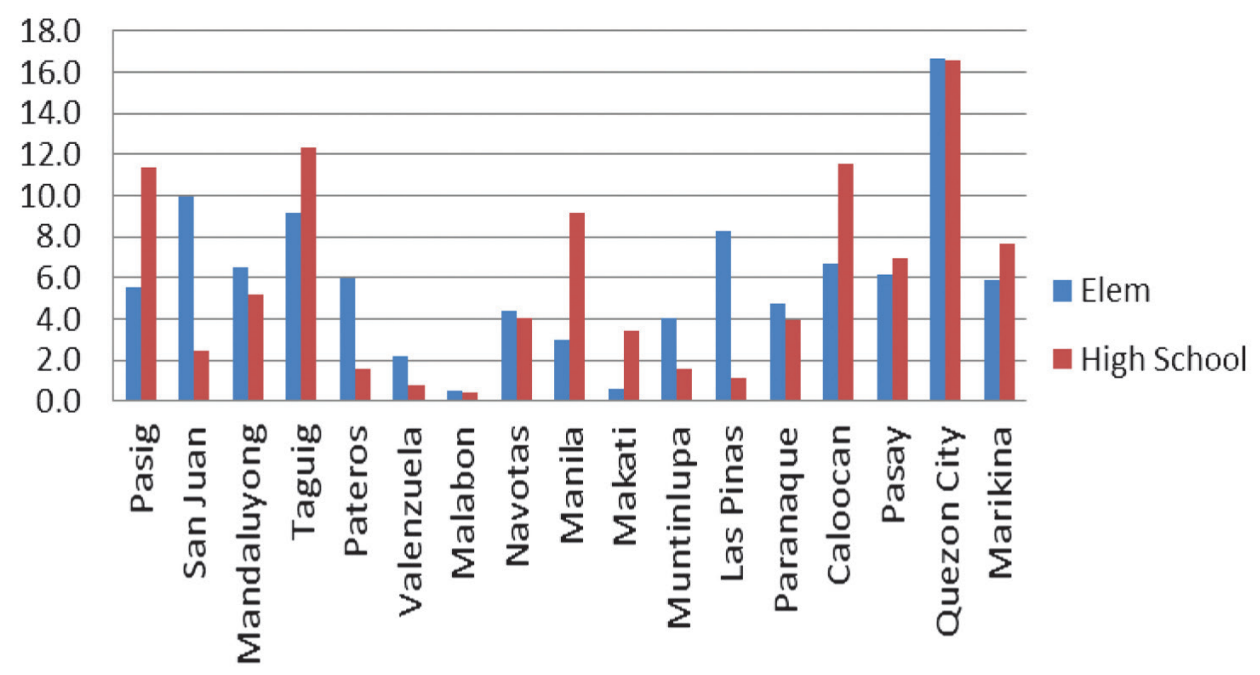

Figure 3. Percent distribution of Aedes spp larvae across cities/municipalities, Metro Manila, November 2013 to February 2014.

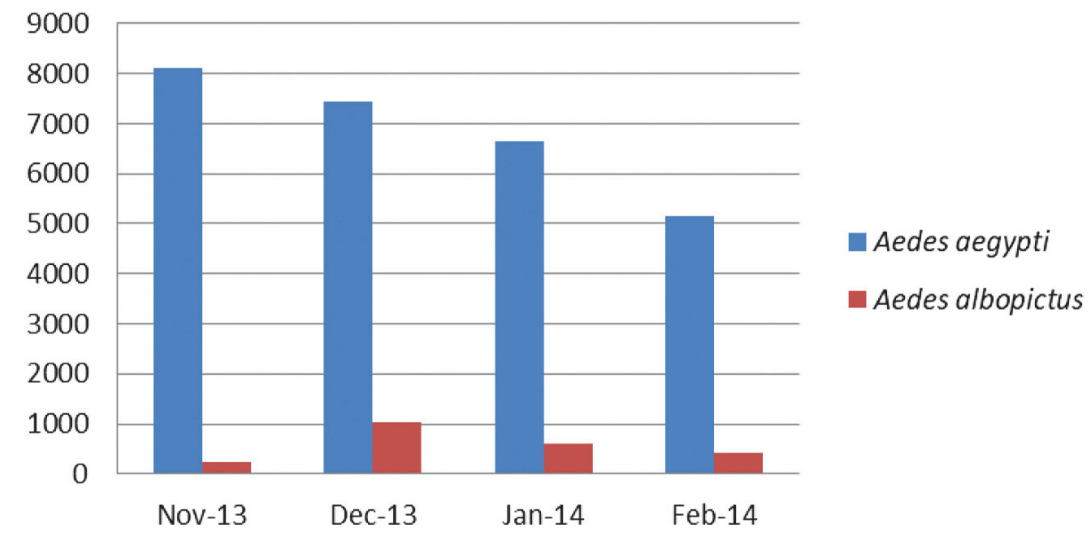

Figure 4. Distribution of Aedes spp larvae across public schools, Metro Manila, November 2013 to February 2014.

and its scope was limited on assessing the mosquito larvae obtained in the ovitraps installed inside the classrooms of public elementary and high schools of Metro Manila from November 2013 to February 2014. A significant difference in the occurrence of mosquito larvae, Aedes aegypti, and Aedes albopictus was observed during the months of collection. In Metro Manila, a type 1 climate is experienced where the months of November to April are considered as the drycool months. ${ }^{11}$ The temperature and available moisture may decrease the mosquito density during the dry-cool months influencing the differences in the occurrence of the mosquito larvae during the period of collection. ${ }^{12}$

The predominance of Aedes aegypti in the ovitraps installed inside the classrooms was evident in the schools examined. No significant differences in the mosquito larvae, Aedes aegypti larvae, and Aedes albopictus larvae collected inside the classrooms of both elementary schools and high schools were evident. A study presents that Aedes aegypti prefers to breed in storage receptacles with clean water. ${ }^{7}$
A study also presented that these mosquitoes mostly favor indoor environments than those of its surrounding. ${ }^{13}$ The coexistence of both Aedes aegypti and Aedes albopictus larvae in the ovitraps in the metropolis poses important threats to the public, especially that these mosquitoes play an important role in the transmission of both the dengue virus and the Chikungunya virus. ${ }^{14,15}$ The distribution of the mosquitoes based on the larvae collected in the ovitraps varied from one city to another. The no-significant difference on the distribution of Aedes aegypti is likely, as the Aedes aegypti larvae were observed in almost all of the ovitraps across all the examined cities and municipalities of Metro Manila. The significant difference on the Aedes albopictus larvae and the total mosquito larvae may be attributable to their distribution and the few occurrence of the larvae compared to that of the Aedes aegypti in the ovitraps. The occurrence of coexistence of Aedes aegypti and Aedes albopictus in the ovitraps was observed in some of the schools. A study supports this result, indicating that the coexistence of Aedes aegypti and 
Aedes albopictus is possible as both vectors could breed in the same containers indoors. ${ }^{13}$ The abundance of Aedes aegypti over Aedes albopictus may be dependent on a variety of factors. Studies present that the environmental conditions and vector control measures instituted ${ }^{13,16}$ predispose the distribution of mosquito larvae in the area. The coexistence of both mosquito larvae may be because both mosquitoes are anthropophilic, day biting and the female mosquitoes lay eggs in containers that may be damp or moist in and around human settlements. ${ }^{17}$ The study was able to demonstrate the utility of the ovitraps as mosquito surveillance tool, concurring with other mosquito surveillance studies indicating that the use of ovitraps is effective in detecting the occurrence of mosquito populations at low infestation levels and at different geographic locations. ${ }^{8,18,19}$

\section{CONCLUSION}

This study assessed the species composition of mosquitoes thriving in ovitraps installed indoors of public elementary and high schools of Metro Manila. Aedes aegypti was the most abundant larva observed in the paddles and water in the examined ovitraps. No single infestation of Aedes albopictus in the examined ovitraps was seen. The coexistence of both Aedes aegypti and Aedes albopictus in some ovitraps were observed in some schools of Metro Manila. Further research should examine the conditions that facilitated the coexistence or competition of mosquito larvae in breeding habitats, as this provides a more detailed analysis of understanding the distribution of vectors and the transmission of infectious diseases among the general populace.

\section{Acknowledgments}

This study was supported by the Philippine Council for Health Research and Development (PCHRD), Department of Science and Technology (DOST). We are grateful to PCHRD-DOST under the leadership of Dr. Jaime Montoya, and the University of the Philippines Los Baños Foundation Inc. College, Laguna for providing the administrative assistance.

\section{Statement of Authorship}

All authors approved the final version submitted.

\section{Author Disclosure}

All authors declared no conflict of interest.

\section{Funding Source}

This paper was funded by the Philippine Council for Health Research and Development, Department of Science and Technology (PCHRD-DOST).

\section{REFERENCES}

1. Dash AP, Bhatia R, Kalra NL. Dengue in South-East Asia: An appraisal of case management and vector control. Dengue Bull. 2013; 36:1-13.

2. National Epidemiologic Center-Department of Health. Disease surveillance report. Dengue Cases Morbidity Week. 2013a; 52:1-5.

3. National Epidemiologic Center-Department of Health. Disease surveillance report. Chikungunya Cases in the Philippines Update. 2013b; 4:1-3.

4. Su GL. Correlation of climatic factors and dengue incidence in Metro Manila, Philippines. Ambio. 2008 Jun; 37 (4): 292-4.

5. Edillo F, Madarieta S. Trends of dengue infections (1997-2008) in Cebu Province, Philippines. Dengue Bull. 2012 Dec; 36:49-61.

6. Bravo LC, Roque Jr. VG, Brett J, Dizon R, L'Azou M. Epidemiology of dengue disease in the Philippines (2000-2011): A systematic literature review. PLoS Negl Trop Dis. 2014 Nov; 8 (11):e3027.

7. Su G, Beronilla A, Yao K. Water quality and Aedes larval mosquito abundance in Caloocan City, Philippines. Dengue Bull. 2012 Dec; 36:187-93.

8. De Las Llagas LA, Bertuso AG, Mistica MS, Samaniego JB. Field evaluation of ovitraps with Piper nigrum to assess its larvicidal and ovipositional effects on dengue mosquito vectors. Philipp Entomologist. 2012; 26 (2):156-75.

9. Rueda LM. Pictorial keys for the identification of mosquitoes (Diptera: Culicidae) associated with dengue virus transmission. Zootaxa. Auckland, New Zealand: Magnolia Press; 2004. pp.1-60.

10. Rashid S, Shahzad F. Levels of serum transaminases in patients of dengue fever during the 2011 outbreak in Lahore, Pakistan. Dengue Bull. 2012; 36:116-23.

11. Flores JF, Balagot VF. Climate of the Philippines. In: Arakawa H, ed. World Survey of Climatology, Vol. 8 Climates of Northern and Eastern Asia. Amsterdam: Elsevier; 1969.

12. Schultz GW. Seasonal abundance of dengue vectors in Manila, Republic of the Philippines. Southeast Asian J Trop Med Public Health. 1993 Jun; 24 (2):369-75.

13. Guo YH, Lai SJ, Huang Q, Ren DS, Zou JH, Liu QY, et al. Coexistence of Aedes aegypti and Aedes albopictus in Jinghong City, Yunnan Province: A survey of Aedes aegypti invasion. J Trop Dis. 2016; 4:227.

14. Reiskind MH, Pesko K, Westbrook CJ, Mores CN. Susceptibility of Florida mosquitoes to infection with Chikungunya virus. Am J Trop Med Hyg. 2008 Mar; 78 (3):422-5.

15. Vazeille M, Moutieller S, Pages F, Jarjaval F, Failloux AB. Introduction of Aedes albopictus in Gabon: What consequences for dengue and Chikungunya transmission? Trop Med Int Health. 2008 Sep; 13 (9):1176-9.

16. Takken W, Geene R, Adam W, Jetten TH, Van der Velden JA. Distribution and dynamics of larval populations of Anopheles messae and A. atroparvus in the Delta of the Rivers Rhine and Meuse, The Netherlands. Ambio. 2002 May; 31 (3):212-18.

17. De Las Llagas LA, Tyagi BK, Bersales LG. Aedes dengue vector ovitrap surveillance system: a framework for Mosquito density prediction. Southeast Asian J Trop Med Public Health. 2016; 47 (4):712-8.

18. Wan-Norafikah O, Nazni W A, Noramiza S, Shafa'ar-Ko'Oohar S, Heah SK, Nor Azlina AH, et al. Ovitrap surveillance and mixed infestation of Aedes aegypti (Linnaeus) and Aedes albopictus (Skuse) in Northern Region and Southern region of Malaysia. Health and the Environment Journal. 2011; 2 (1):1-5.

19. Mistica MS, De LasLlagas LA, Bertuso AG. Dengue mosquito ovitrapping and preventive fogging trials in the Philippines. Philipp Entomologist. 2007; 21 (2):136-45. 